\title{
Constraints on the infrared behavior of the ghost propagator in Yang-Mills theories
}

\author{
A. Cucchieri \\ Instituto de Física de São Carlos, Universidade de São Paulo, Caixa Postal 369, 13560-970 São Carlos, SP, Brazil \\ T. Mendes* \\ DESY-Zeuthen, Platanenallee 6, 15738 Zeuthen, Germany
}

(Received 17 April 2008; published 7 November 2008)

\begin{abstract}
We present rigorous upper and lower bounds for the momentum-space ghost propagator $G(p)$ of YangMills theories in terms of the smallest nonzero eigenvalue (and of the corresponding eigenvector) of the Faddeev-Popov matrix. We apply our analysis to data from simulations of SU(2) lattice gauge theory in Landau gauge, using the largest lattice sizes to date. Our results suggest that, in three and in four spacetime dimensions, the Landau gauge ghost propagator is not enhanced as compared to its tree-level behavior. This is also seen in plots and fits of the ghost dressing function. In the two-dimensional case, on the other hand, we find that $G(p)$ diverges as $p^{-2-2 \kappa}$ with $\kappa \approx 0.15$, in agreement with A. Maas, Phys. Rev. D 75, 116004 (2007). We note that our discussion is general, although we make an application only to pure gauge theory in Landau gauge. Our simulations have been performed on the IBM supercomputer at the University of São Paulo.
\end{abstract}

DOI: 10.1103/PhysRevD.78.094503

PACS numbers: 11.15.Ha, 12.38.Aw, 14.70.Dj

\section{INTRODUCTION}

In the Gribov-Zwanziger scenario [1], confinement of quarks (in Landau and in Coulomb gauge) is related to a ghost propagator $G(p)$ enhanced in the infrared (IR) limit when compared to the tree-level propagator $1 / p^{2}$. An enhanced ghost propagator is also required by the KugoOjima criterion [2] in order to explain confinement of color charge (in Landau gauge).

In order to investigate the origin of this IR enhancement we consider, following Ref. [3], a generic gauge condition $\mathcal{F}[A]=0$, where $A$ is the gauge field. The condition is imposed on the lattice by minimizing a functional $E[U]$, where $U$ is the (gauge) link variable. From the second variation of $E[U]$ one can define the Faddeev-Popov (FP) matrix $\mathcal{M}(a, x ; b, y)$. (Here we use $a$ and $b$ to indicate color indices, whereas $x$ and $y$ represent points of the lattice.) In the $\mathrm{SU}\left(N_{c}\right)$ case, $\mathcal{M}$ is an $\left(N_{c}^{2}-1\right) V \times\left(N_{c}^{2}-\right.$ 1) $V$ matrix, where $V=N^{d}$ is the lattice volume, given by the number of points $N$ along each side of the lattice and by the space-time dimension $d$. This matrix is real and symmetric with respect to the exchange $(a, x) \leftrightarrow(b, y)$ (see Ref. [4] for a thorough discussion of the properties of the FP matrix in Landau gauge). At any (local) minimum of $E[U]$, all the eigenvalues of $\mathcal{M}$ are positive (modulo trivial null eigenvalues). The set of all minima of $E[U]$ is the socalled Gribov region $\Omega$. On the boundary of $\Omega$ - known as the first Gribov horizon $\partial \Omega$-the smallest nontrivial eigenvalue $\lambda_{\text {min }}$ of the FP matrix is null. Since the configuration space has very large dimensionality we expect that,

\footnotetext{
*Permanent address: Instituto de Física de São Carlos, Universidade de São Paulo, C.P. 369, 13560-970 São Carlos, SP, Brazil.
}

in the limit of large lattice volumes $V$, entropy favors configurations near $\partial \Omega$ [5-7], i.e. $\lambda_{\min }$ should go to zero in this limit. This is indeed the case in $2 \mathrm{~d}$ [8], 3d [9], and $4 \mathrm{~d}$ Landau gauge [10], in $4 d$ Coulomb gauge [7], and in $4 d$ maximally Abelian gauge [11].

The ghost propagator is written in terms of the inverse of the FP matrix as

$$
G(p)=\frac{1}{N_{c}^{2}-1} \sum_{x, y, a} \frac{e^{-2 \pi i k \cdot(x-y)}}{V} \mathcal{M}^{-1}(a, x ; a, y),
$$

where the lattice momentum $p(k)$ has components $p_{\mu}(k)=2 \sin \left(\pi k_{\mu} / N\right)$ with $k_{\mu}=0,1, \ldots, N-1$. Also, here and in the formulas below, a path-integral average is understood. Since $\mathcal{M}(a, x ; b, y)$ develops a null eigenvalue at the Gribov horizon $\partial \Omega$, one might expect that the corresponding ghost propagator diverges at small momenta in the infinite-volume limit. This could in turn introduce a long-range effect in the theory, related to the colorconfinement mechanism [5,7]. Indeed, in several numerical studies (using relatively small lattice volumes), the ghost propagator $G(p)$ seems to be IR-enhanced in 3d [9] and $4 \mathrm{~d}$ Landau gauge $[12,13]$ and in $4 \mathrm{~d}$ Coulomb gauge [14]. On the other hand, in maximally Abelian gauge [11] one finds an IR-finite $G(p)$. At the same time, recent results in Landau gauge using very large lattice volumes [15-17] suggest an essentially tree-level-like IR ghost propagator $G(p)$ in $3 \mathrm{~d}$ and in $4 \mathrm{~d}$. (A similar result has been obtained in Refs. [18,19] using different analytic approaches.) Finally, in the $2 \mathrm{~d}$ case, one finds [8] $G(p) \sim p^{-2.3}$ after extrapolating data for the IR exponent $\kappa$ to infinite volume. Therefore, we have cases for which $G(p)$ is not IRenhanced in the infinite-volume limit and thus the argument reported above cannot be valid in general [3]. The 
aim of this work is to understand under what conditions one should expect an IR-enhanced ghost propagator $G(p)$. This is done by investigating constraints on the behavior of $G(p)$ and thus obtaining better control over its infinitevolume extrapolation. Clearly, since the only diverging quantity (besides the lattice volume) is $1 / \lambda_{\min }$, the IR enhancement of $G(p)$ may be closely related to this eigenvalue and to the projection of its corresponding eigenvector $\psi_{\min }(a, x)$ on the plane waves $e^{-2 \pi i k \cdot x} / \sqrt{V}$ for small momenta $p(k)$.

\section{LOWER AND UPPER BOUNDS FOR $G(p)$}

In this section we obtain upper and lower bounds for the momentum-space ghost propagator $G(p)$ in terms of the smallest nonzero eigenvalue $\lambda_{\min }$ [and of the corresponding eigenvector $\left.\psi_{\min }(a, x)\right]$ of the FP matrix $\mathcal{M}(a, x ; b, y)$. To this end, let us first introduce our notation. As usual, we define the generic eigenvalue $\lambda_{i}$ of $\mathcal{M}(a, x ; b, y)$ and the corresponding eigenvector $\psi_{i}(a, x)$ by using the relation $\mathcal{M}(a, x ; b, y) \psi_{i}(b, y)=\lambda_{i} \psi_{i}(a, x)$, where the index $i$ takes values $1,2, \ldots,\left(N_{c}^{2}-1\right) V$ and the sum over repeated indices is understood. As stressed in the Introduction, this matrix is positive (or semipositive) definite, whenever we use a minimizing condition in order to fix a gauge (minimal gauge). Also, since it is obtained from a second-order expansion, this matrix can always be written in a symmetric form. Then, the eigenvectors $\psi_{i}(a, x)$ can be assumed orthogonal to each other and normalized as $\sum_{a, x} \psi_{i}(a, x) \psi_{j}(a, x)^{*}=\delta_{i j}$, where $*$ indicates complex conjugation. Using this notation we can write

$$
\mathcal{M}(a, x ; b, y)=\sum_{i, \lambda_{i} \neq 0} \lambda_{i} \psi_{i}(a, x) \psi_{i}(b, y)^{*} .
$$

Note that we are working in the space orthogonal to the kernel of the FP matrix. Then we can write the inverse of this matrix as [20]

$$
\mathcal{M}^{-1}(a, x ; b, y)=\sum_{i, \lambda_{i} \neq 0} \lambda_{i}^{-1} \psi_{i}(a, x) \psi_{i}(b, y)^{*} .
$$

By using Eq. (1) we obtain

$$
G(p)=\frac{1}{N_{c}^{2}-1} \sum_{i, \lambda_{i} \neq 0} \sum_{a} \lambda_{i}^{-1}\left|\tilde{\psi}_{i}(a, p)\right|^{2},
$$

where $\tilde{\psi}_{i}(a, p)=\sum_{x} \psi_{i}(a, x) e^{-2 \pi i k \cdot x} / \sqrt{V}$ is the Fourier transform of the eigenvector $\psi_{i}(a, x)$. Since all the nonzero eigenvalues are positive and $0<\lambda_{\min } \leq \lambda_{i}$, we can write the inequalities

$$
\begin{gathered}
\frac{1}{N_{c}^{2}-1} \frac{1}{\lambda_{\min }} \sum_{a}\left|\tilde{\psi}_{\min }(a, p)\right|^{2} \leq G(p), \\
G(p) \leq \frac{1}{N_{c}^{2}-1} \frac{1}{\lambda_{\min }} \sum_{i, \lambda_{i} \neq 0} \sum_{a}\left|\tilde{\psi}_{i}(a, p)\right|^{2} .
\end{gathered}
$$

(Note that we assume nondegenerate eigenvalues.
However, the second inequality applies also when $\lambda_{\min }$ is degenerate and the first one can be easily modified to take a degeneracy into account.) By summing and subtracting in Eq. (6) the contributions from the eigenvectors corresponding to a null eigenvalue and using the completeness relation $\sum_{i} \psi_{i}(a, x) \psi_{i}(b, y)^{*}=\delta_{a b} \delta_{x y}$, we find

$$
G(p) \leq \frac{1}{\lambda_{\min }}\left[1-\frac{1}{N_{c}^{2}-1} \sum_{j, \lambda_{j}=0} \sum_{a}\left|\tilde{\psi}_{j}(a, p)\right|^{2}\right] .
$$

Let us stress that the above results are simply a consequence of the positivity of the FP matrix, i.e. they apply to gauge-fixed configurations that belong to the interior of the first Gribov region.

\section{BOUNDS IN LANDAU GAUGE}

In the Landau case the eigenvectors corresponding to the zero eigenvalue are constant modes, i.e. they contribute only to the case $p=0$. Thus, for any nonzero momentum we have

$$
\frac{1}{N_{c}^{2}-1} \frac{1}{\lambda_{\min }} \sum_{a}\left|\tilde{\psi}_{\min }(a, p)\right|^{2} \leq G(p) \leq \frac{1}{\lambda_{\min }} .
$$

As said in the Introduction, in the infinite-volume limit the measure gets concentrated on the boundary of the (first) Gribov region, i.e. $\lambda_{\min }$ goes to zero in this limit. In particular, as for the Laplacian operator, one can expect to find $\lambda_{\text {min }} \sim N^{-\alpha}$ for large $N$. Now, if we make the hypothesis of a power-law behavior $p^{-2-2 \kappa}$ for $G(p)$ in the IR limit and consider the smallest nonzero momentum on the lattice [i.e. $p_{s}=2 \sin (\pi / N)$ ], we have $G\left(p_{s}\right) \sim$ $N^{2+2 \kappa}$ in the limit of large $N$. Then from Eq. (8) we get $2+2 \kappa \leq \alpha$. Thus, assuming a power-law behavior for $G(p), \alpha>2$ is a necessary condition to obtain a ghost propagator $G(p)$ enhanced in the IR limit compared to the tree-level behavior $1 / p^{2}$.

The lower bound in Eq. (8) clearly depends on the behavior of the quantity $\left|\tilde{\psi}_{\min }\left(a, p_{s}\right)\right|^{2}$. Note that this quantity cannot diverge in the infinite-volume limit. (Actually, since the eigenvectors are normalized, this quantity is always between 0 and 1.) Thus, if we furthermore make the assumption of a behavior at large $N$ given by $N^{-\gamma}$, with $\gamma \geq 0$, we find the condition $\alpha-\gamma \leq 2+2 \kappa$.

As a check of the proposed bounds, we collect here results for the ghost propagator $G\left(p_{s}\right)$ at the smallest nonzero momentum $p_{s}=2 \sin (\pi / N)$ and for the smallest nonzero eigenvalue $\lambda_{\min }$ from Refs. [6,9,12,15] for the $3 d$ and $4 \mathrm{~d}$ cases. These data are shown for the smaller lattices in Tables I and II and for the larger lattices in Fig. 1. We also present new data in the two-dimensional case. (These data were obtained together with the data for the gluon propagator reported in [21].) All data refer to the $\mathrm{SU}(2)$ case. Let us recall that recent studies $[17,22]$ have verified the analytic prediction that Landau gauge gluon and ghost propagators in $\mathrm{SU}(2)$ and in $\mathrm{SU}(3)$ are rather similar. Thus, 
TABLE I. The ghost propagator $G\left(p_{s}\right)$ for the smallest nonzero momentum $p_{s}=2 \sin (\pi / N)$ and the inverse of the smallest nonzero eigenvalue $\lambda_{\min }$ of the FP matrix for various lattice volumes and $\beta$ values in the $3 \mathrm{~d}$ case. Data (in physical units) are taken from Ref. [9].

\begin{tabular}{ccccc}
\hline \hline$N$ & $\beta$ & $a^{-1}[\mathrm{GeV}]$ & $G\left(p_{s}\right)\left[\mathrm{GeV}^{-2}\right]$ & $1 / \lambda_{\min }\left[\mathrm{GeV}^{-2}\right]$ \\
\hline 20 & 6.0 & 1.733 & $7.3(1)$ & $32.5(7)$ \\
30 & 6.0 & 1.733 & $19.4(3)$ & $94(2)$ \\
20 & 4.2 & 1.136 & $21.0(4)$ & $107(3)$ \\
30 & 4.2 & 1.136 & $54.5(8)$ & $282(6)$ \\
\hline \hline
\end{tabular}

we expect that the analysis presented here should apply also to the $S U(3)$ case. In all cases the quantity $1 / \lambda_{\min }$ has been evaluated using the average value for $\lambda_{\min }$ and propagation of errors.

As one can see, the upper bound in Eq. (8) is always satisfied. (Of course, this is the case also when $p \neq p_{s}$.) The bound has also been verified using the data at $\beta=0$ from Refs. [6,12]. We have fitted the data for $G\left(p_{s}\right)$ and for $\lambda_{\min }$ as a function of $L$ for the data shown in Fig. 1 using a power-law Ansatz. It is interesting to note that (in $2 \mathrm{~d}$ and in 4d) we actually find $\alpha$ smaller than $2+2 \kappa$. The bound is still satisfied, since the multiplicative constant in the fit of $\lambda_{\min }$ is larger. It would be interesting to see how the upper bound is realized when even larger lattice volumes are considered. Also, note that the upper bound in the $4 d$ case seems to saturate as the volume increases. This would indicate that the contribution to $G(p)$ from all the eigenvalues $\lambda_{i}>\lambda_{\min }$ stays finite at large volume and that the exponent $\gamma$, defined above, is zero. Let us note that this is not the case at smaller lattice volumes, since then one needs to consider the contribution to $G(p)$ from the first 150-200 smaller eigenvalues in order to reproduce the behavior of the propagator at small momenta [10]. Finally, let us recall that finite-size effects for $G(p)$ (at fixed $p$ ) are generally very small. This can be explained considering the spectral density of the FP matrix, as done in Ref. [3]. Thus, our results indicate that in $3 \mathrm{~d}$ and in $4 \mathrm{~d}$ the ghost propagator is likely not IR-enhanced, while in $2 \mathrm{~d}$

TABLE II. As in Table I for the $4 d$ case. Data (in lattice units) are taken from Refs. [6,12] using the average called first copy. We find similar results when considering data obtained using the average called absolute minimum.

\begin{tabular}{lccc}
\hline \hline$N$ & $\beta$ & $G\left(p_{s}\right)$ & $1 / \lambda_{\min }$ \\
\hline 8 & 0.8 & $8.94(8)$ & $91(3)$ \\
12 & 0.8 & $22.1(6)$ & $210(20)$ \\
16 & 0.8 & $40.6(7)$ & $360(60)$ \\
8 & 1.6 & $7.2(1)$ & $61(3)$ \\
16 & 1.6 & $32.1(3)$ & $220(20)$ \\
8 & 2.7 & $3.4(1)$ & $11.0(4)$ \\
12 & 2.7 & $7.1(3)$ & $25(1)$ \\
16 & 2.7 & $12.9(6)$ & $45(4)$ \\
\hline \hline
\end{tabular}
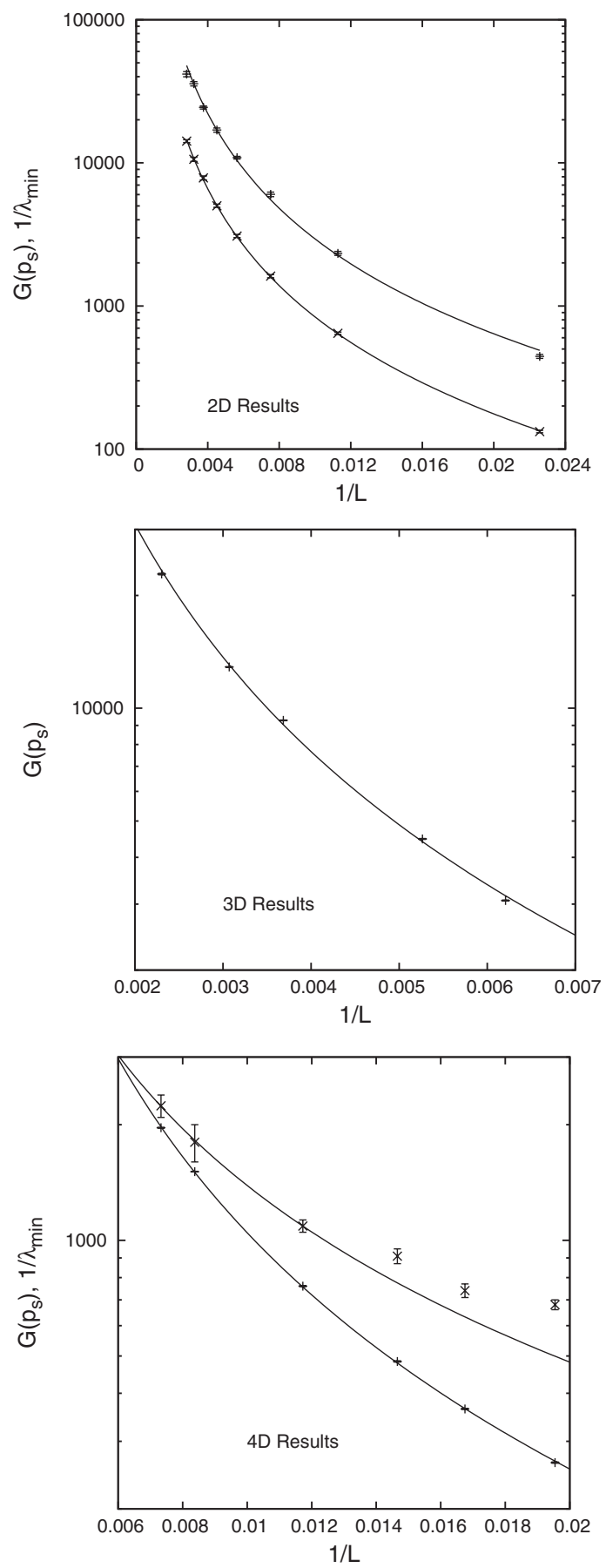

FIG. 1. The ghost propagator $G\left(p_{s}\right)$ for the smallest nonzero momentum $p_{s}=2 \sin (\pi / N)$ (in $\mathrm{GeV}^{-2}$ ) as a function of the inverse lattice side $1 / L(\mathrm{GeV})$ for the $2 \mathrm{~d}$ case (top, at $\beta=10$ with volumes up to $320^{2}$ ), $3 \mathrm{~d}$ case (center, at $\beta=3$, with volumes up to $320^{3}$ ), and the $4 \mathrm{~d}$ case (bottom, at $\beta=2.2$ with volumes up to $128^{4}$ ). Data are taken from Ref. [15] for the $3 d$ and the $4 d$ cases. We also show (in $2 d$ and in $4 d$ ) the inverse of the smallest nonzero eigenvalue $\lambda_{\min }$ of the FP matrix (in $\mathrm{GeV}^{-2}$ ). In these two cases one can verify the inequality $1 / \lambda_{\min } \geq G\left(p_{s}\right)$. The fitting parameters are reported in Table III. Note that we did not evaluate $\lambda_{\text {min }}$ for all our configurations. 
TABLE III. Fits of $G\left(p_{s}\right)$ and of $1 / \lambda_{\min }$, respectively, using the Ansätze $b L^{2+2 \kappa}$ and $c L^{\alpha}$. In the $4 \mathrm{~d}$ case the fit for $1 / \lambda_{\min }$ has been done by considering only the three largest physical volumes. When considering the three smallest physical volumes we find $\alpha=0.9(3)$. In the other cases all data have been considered for the fit.

\begin{tabular}{lllcc}
\hline \hline$d$ & \multicolumn{1}{c}{$b$} & $2+2 \kappa$ & $c$ & $\alpha$ \\
\hline 2 & $0.026(1)$ & $2.251(9)$ & $0.12(3)$ & $2.20(4)$ \\
3 & $0.11(3)$ & $2.02(4)$ & & \\
4 & $0.086(3)$ & $2.043(8)$ & $1.2(1)$ & $1.53(2)$ \\
\hline \hline
\end{tabular}
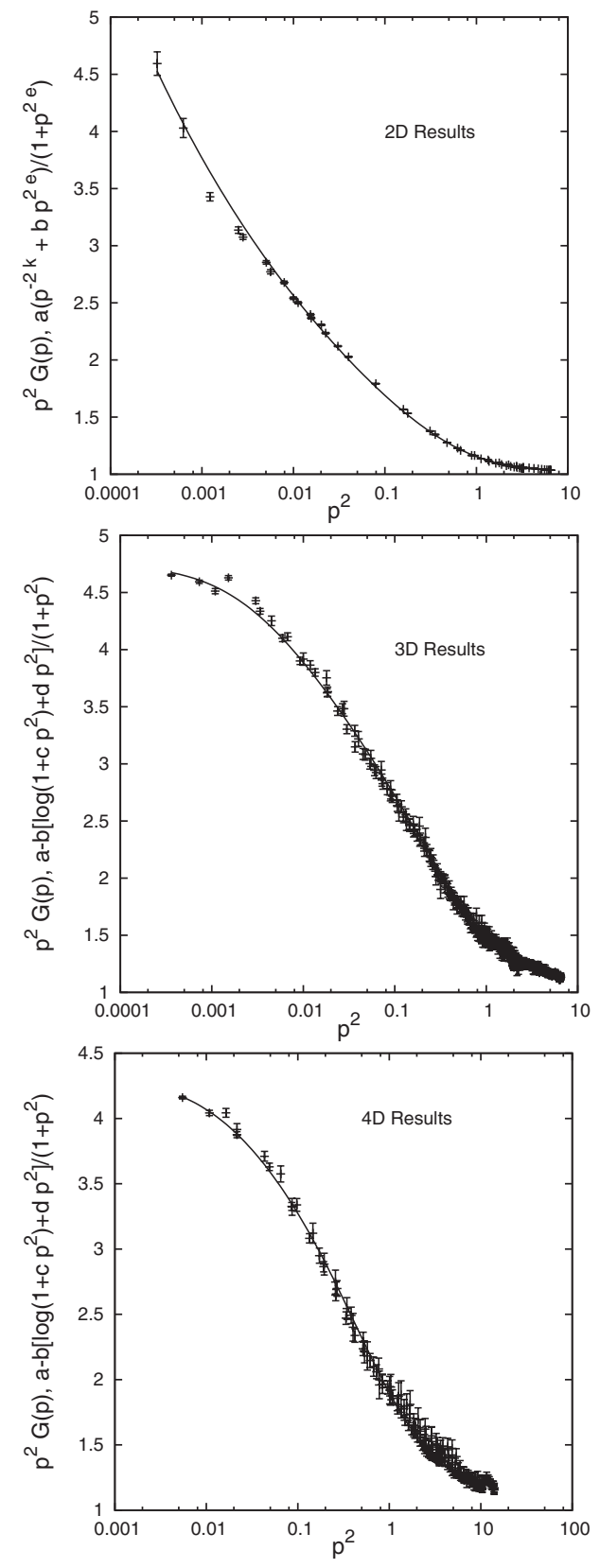

FIG. 2. The ghost dressing function $p^{2} G\left(p^{2}\right)$ as a function of $p^{2}$ (in $\mathrm{GeV}$ ) for the $2 \mathrm{~d}$ case (top, at $\beta=10$ with volume $320^{2}$ ), $3 \mathrm{~d}$ case (center, at $\beta=3$, with volume $240^{3}$ ), and the $4 \mathrm{~d}$ case (bottom, at $\beta=2.2$ with volume $80^{4}$ ). The fitting functions and the corresponding fitting parameters are reported in Table V.
TABLE IV. Fits of $p^{2} G\left(p^{2}\right)$ using the Ansätze $a p^{-2 \kappa}$ in the $2 \mathrm{~d}$ case and $a-b \log \left(1+c p^{2}\right)$ in the $3 \mathrm{~d}$ and $4 \mathrm{~d}$ cases. In the first case we used the data in the range $p^{2} \in[0,0.5]$ for the fit. In the other two cases the data in the range $p^{2} \in[0,1]$ have been considered.

\begin{tabular}{llllc}
\hline \hline$d$ & \multicolumn{1}{c}{$a$} & \multicolumn{1}{c}{$\kappa$} & \multicolumn{1}{c}{$b$} & $c$ \\
\hline 2 & $1.134(7)$ & $0.177(2)$ & & \\
3 & $4.7(1)$ & & $0.579(5)$ & $320(20)$ \\
4 & $4.28(1)$ & & $0.69(2)$ & $33(3)$ \\
\hline \hline
\end{tabular}

TABLE V. Fits of $p^{2} G\left(p^{2}\right)$ using the Ansätze $a\left(p^{-2 k}+\right.$ $\left.b p^{2 e}\right) /\left(1+p^{2 e}\right)$ in the $2 \mathrm{~d}$ case and $a-b\left[\log \left(1+c p^{2}\right)+\right.$ $\left.d p^{2}\right] /\left(1+p^{2}\right)$ in the $3 \mathrm{~d}$ and $4 \mathrm{~d}$ cases. In the three cases we used the whole range of momenta for the fit.

\begin{tabular}{lcccccc}
\hline \hline$d$ & $a$ & $\kappa$ & \multicolumn{1}{c}{$b$} & \multicolumn{1}{c}{$e$} & $c$ & $d$ \\
\hline 2 & $1.24(3)$ & $0.16(2)$ & $0.86(3)$ & $0.75(15)$ & & \\
3 & $4.75(1)$ & & $0.491(5)$ & & $450(30)$ & $7.1(1)$ \\
4 & $4.32(2)$ & & $0.38(1)$ & & $80(10)$ & $8.2(3)$ \\
\hline \hline
\end{tabular}

we obtain $\kappa \approx 0.1$. We note that our result in the $2 \mathrm{~d}$ case is essentially in agreement with Ref. [8], where however the value of $\kappa$ is obtained by first fitting $G(p)$ in an appropriate momentum range for each lattice volume and then performing the extrapolation to infinite volume by a fourparameter fit. The fits for $\lambda_{\min }$ and $G\left(p_{s}\right)$ as a function of $L$ are usually simpler, as shown above.

These results are confirmed if one considers the dressing function $p^{2} G\left(p^{2}\right)$ at the largest lattice volumes for the three cases (see Fig. 2). Indeed, the data in the $2 \mathrm{~d}$ case can be fitted using a power-law Ansatz $a p^{-2 \kappa}$ (see Table IV), with $\kappa=0.177(2)$, essentially in agreement with [8]. The fitted value for $\kappa$ decreases if one considers fewer points, dropping points with larger $p$, and becomes $0.152(7)$ when considering data with $p^{2} \in[0,0.1]$. The same Ansatz does not work in $3 \mathrm{~d}$ and in $4 \mathrm{~d}$. In these cases the data for the dressing function can be described by the Ansatz $a-$ $b \log \left(1+c p^{2}\right)$, inspired by the logarithmic corrections suggested in Ref. [19]. In both cases one finds (approximately) $p^{2} G\left(p^{2}\right) \rightarrow 4.5$ in the limit $p \rightarrow 0$. This result supports the value $\kappa \approx 0$ obtained above, when considering the bound using $\lambda_{\min }$. It is interesting that one can also obtain a relatively good fit for the dressing function in the whole range of momenta by considering the fitting function $a\left(p^{-2 k}+b p^{2 e}\right) /\left(1+p^{2 e}\right)$ in the $2 \mathrm{~d}$ case and $a-$ $b\left[\log \left(1+c p^{2}\right)+d p^{2}\right] /\left(1+p^{2}\right)$ in $3 \mathrm{~d}$ and in $4 \mathrm{~d}$. These fits and the corresponding parameters are reported in Fig. 2 and in Table V.

\section{CONCLUSIONS}

Our data suggest that the Landau gauge ghost propagator does not diverge faster than $1 / p^{2}$ at small momenta in $3 \mathrm{~d}$ and in $4 \mathrm{~d}$, while in $2 \mathrm{~d} G(p)$ does not diverge faster than $p^{-2-2 \kappa}$ with $\kappa$ between 0.1 and 0.2 , in agreement with 
Ref. [8]. These results have been obtained by considering the inequality in Eq. (7) and by fitting the data for $G\left(p_{s}\right)$ and $\lambda_{\min }$ as a function of $L$. In particular, we note that the use of the upper bound to constrain $G(p)$ is quite convenient, since $\lambda_{\min }$ does not depend on $p$. It might thus be of interest to optimize the evaluation of $\lambda_{\min }$ and of $\psi_{\min }(a, x)$.

The results obtained here in the $3 \mathrm{~d}$ and $4 \mathrm{~d}$ cases, together with those reported in Ref. [21] for the gluon propagator, seem to contradict the Gribov-Zwanziger confinement scenario for Landau gauge. On the other hand, one should establish if the behavior of gluon and ghost propagators at very small momenta is really so essential for the explanation of confinement. After all, when dynamical quarks are considered [23], string breaking should manifest itself at a scale of about a fermi, i.e. for an energy scale of about $200 \mathrm{MeV}$. Thus, for the physics of hadrons the behavior of the propagators at intermediate momenta is probably more important. Let us recall that for a space-time separation of about $1 \mathrm{~F}$ the transverse gluon propagator violates reflection positivity [24], i.e. becomes negative in real-space coordinates, and the exponent $\kappa$ for the ghost propagator is about $0.2-0.3$ considering $p \sim 0.5 \mathrm{GeV}[9,12,13,15-$ 17]. Thus, in the range 200-500 MeV, nonperturbative effects are clearly present in the behavior of the gluon and of the ghost propagator. For this range of momenta the use of our lower bound for the ghost propagator could probably also be valuable. Of course, the open problem is if these effects suffice to explain color confinement.

\section{ACKNOWLEDGMENTS}

Discussions with several participants of the workshop on Quarks and Hadrons in Strong QCD (St. Goar) are acknowledged. We also thank R. Sommer for helpful comments on the manuscript. We acknowledge partial support from FAPESP (under Grant No. 05/59919-7) and from CNPq (including Grants No. 476221/2006-4 and No. 455353/2007-7). The work of T. M. is supported also by the Alexander von Humboldt Foundation. Our simulations were made possible by FAPESP Grant No. 04/089283.
[1] V. N. Gribov, Nucl. Phys. B139, 1 (1978); D. Zwanziger, Nucl. Phys. B412, 657 (1994); Y. L. Dokshitzer and D. E. Kharzeev, Annu. Rev. Nucl. Part. Sci. 54, 487 (2004); R. F. Sobreiro and S.P. Sorella, arXiv:hep-th/0504095; R. Alkofer and J. Greensite, J. Phys. G 34, S3 (2007).

[2] T. Kugo and I. Ojima, Prog. Theor. Phys. Suppl. 66, 1 (1979); Prog. Theor. Phys. 71, 1121(E) (1984); T. Kugo, arXiv:hep-th/9511033.

[3] A. Cucchieri, AIP Conf. Proc. 892, 22 (2007).

[4] A. Cucchieri, T. Mendes, and A. Mihara, Phys. Rev. D 72, 094505 (2005).

[5] D. Zwanziger, Nucl. Phys. B412, 657 (1994); Phys. Rev. Lett. 90, 102001 (2003).

[6] A. Cucchieri, Nucl. Phys. B521, 365 (1998).

[7] J. Greensite, S. Olejnik, and D. Zwanziger, J. High Energy Phys. 05 (2005) 070.

[8] A. Maas, Phys. Rev. D 75, 116004 (2007).

[9] A. Cucchieri, A. Maas, and T. Mendes, Phys. Rev. D 74, 014503 (2006).

[10] A. Sternbeck, E. M. Ilgenfritz, and M. Muller-Preussker, Phys. Rev. D 73, 014502 (2006).

[11] T. Mendes, A. Cucchieri, and A. Mihara, AIP Conf. Proc. 892, 203 (2007).

[12] A. Cucchieri, Nucl. Phys. B508, 353 (1997).

[13] S. Furui and H. Nakajima, Phys. Rev. D 69, 074505 (2004); J. C. R. Bloch, A. Cucchieri, K. Langfeld, and T. Mendes, Nucl. Phys. B687, 76 (2004); J. Gattnar, K. Langfeld, and H. Reinhardt, Phys. Rev. Lett. 93, 061601 (2004); A. Sternbeck, E. M. Ilgenfritz, M. MuellerPreussker, and A. Schiller, Phys. Rev. D 72, 014507 (2005); O. Oliveira and P. J. Silva, Braz. J. Phys. 37, 201
(2007); A. Cucchieri and T. Mendes, Phys. Rev. D 73, 071502 (2006).

[14] K. Langfeld and L. Moyaerts, Phys. Rev. D 70, 074507 (2004).

[15] A. Cucchieri and T. Mendes, Proc. Sci., LATTICE2007 (2007) 297.

[16] I. L. Bogolubsky, E. M. Ilgenfritz, M. Muller-Preussker, and A. Sternbeck, Proc. Sci., LATTICE2007 (2007) 290; O. Oliveira and P. J. Silva, arXiv:0705.0964.

[17] A. Sternbeck, L. von Smekal, D. B. Leinweber, and A. G. Williams, Proc. Sci., LATTICE2007 (2007) 340.

[18] A. C. Aguilar and A. A. Natale, J. High Energy Phys. 08 (2004) 057; M. Frasca, arXiv:0709.2042; D. Dudal, S. P. Sorella, N. Vandersickel, and H. Verschelde, Phys. Rev. D 77, 071501 (2008); D. Dudal et al., Phys. Rev. D 78, 065047 (2008).

[19] Ph. Boucaud et al., J. High Energy Phys. 06 (2008) 012; 06 (2008) 099.

[20] In the theory of Green's functions this quantity is known as the pseudo Green's function of $\mathcal{M}(a, x ; b, y)$ [see, for example, G. Barton, Elements of Green's Functions and Propagation Potentials, Diffusion, and Waves (Oxford University Press, New York, 1989)].

[21] A. Cucchieri and T. Mendes, Phys. Rev. Lett. 100, 241601 (2008).

[22] A. Cucchieri, T. Mendes, O. Oliveira, and P. J. Silva, Phys. Rev. D 76, 114507 (2007); A. Maas and S. Olejnik, J. High Energy Phys. 02 (2008) 070.

[23] Simulations in the unquenched case [25] have shown that the behavior of the ghost propagator is essentially not affected compared to the quenched case. 
[24] K. Langfeld, H. Reinhardt, and J. Gattnar, Nucl. Phys. B621, 131 (2002); A. Cucchieri, T. Mendes, and A. R. Taurines, Phys. Rev. D 71, 051902 (2005); P. J. Silva and O. Oliveira, Proc. Sci., LAT2006 (2006) 075; A. Sternbeck et al., Proc. Sci., LAT2006 (2006) 076; P. O. Bowman et al., Phys. Rev. D 76, 094505 (2007).

[25] E. M. Ilgenfritz et al., Braz. J. Phys. 37, 193 (2007); S. Furui and H. Nakajima, Phys. Rev. D 73, 094506 (2006). 